\title{
Proteomics-based Multimarker Model Improves Neurofilament Light Chain for Predicting Neurological Outcome after Cardiac Arrest
}

\section{Raphael Wurm}

Medical University of Vienna https://orcid.org/0000-0003-3027-7775

Henrike Arfsten

Medical University of Vienna: Medizinische Universitat Wien

\section{Besnik Muqaku}

University of Vienna Faculty of Chemistry: Universitat Wien Fakultat fur Chemie

\section{Markus Ponleitner}

Medical University of Vienna Department of Neurology: Medizinische Universitat Wien Universitatsklinik fur Neurologie

\section{Andrea Bileck}

University of Vienna Faculty of Chemistry: Universitat Wien Fakultat fur Chemie

\section{Patrick Altmann}

Medical University of Vienna Department of Neurology: Medizinische Universitat Wien Universitatsklinik fur Neurologie

\section{Paulus Rommer}

Medical University of Vienna Department of Neurology: Medizinische Universitat Wien Universitatsklinik fur Neurologie

\section{Stefan Seidel}

Medical University of Vienna Department of Neurology: Medizinische Universitat Wien Universitatsklinik fur Neurologie

\section{Pia Hubner}

Medical University of Vienna Department of Emergency Medicine: Medizinische Universitat Wien Universitatsklinik fur Notfallmedizin

\section{Fritz Sterz}

Medical University of Vienna Department of Emergency Medicine: Medizinische Universitat Wien Universitatsklinik fur Notfallmedizin

\section{Gottfried Heinz}

Medical University of Vienna: Medizinische Universitat Wien

\section{Christopher Gerner}

University of Vienna Faculty of Chemistry: Universitat Wien Fakultat fur Chemie Christopher Adlbrecht ( $\sim$ c.adlbrecht@imed19.at) 
Imed 19, private clinical research center

Klaus Distelmaier

Medical University of Vienna: Medizinische Universitat Wien

\section{Research}

Keywords: Cardiac arrest, cardiopulmonary resuscitation, prognostication, proteomics, neurofilament light chain

Posted Date: November 19th, 2020

DOl: https://doi.org/10.21203/rs.3.rs-108273/v1

License: (c) (i) This work is licensed under a Creative Commons Attribution 4.0 International License. Read Full License 


\section{Abstract}

Background:

Out of hospital cardiac arrest (OHCA) is a life-threatening event. Continuous advances in management increased initial survival, but the rate of favorable neurological outcome remains low. We have previously shown the usefulness of proteomics to identify novel biomarkers to predict this outcome. Neurofilament light chain ( $\mathrm{NfL}$ ), a marker of axonal damage, has since emerged as a promising single marker. The aim of this study was thus to assess the predictive value of $\mathrm{NfL}$ and compare it to our established model.

Methods:

$\mathrm{NfL}$ was measured in plasma samples from OHCA drawn at 48 hours after the event using single molecule assays. Neurological function at discharge from ICU was recorded on the cerebral performance category (CPC) scale. Predictive ability was assessed for $\mathrm{NfL}$ and compared to an established multimarker model.

Results:

Seventy patients were included into this analysis, of whom 21 (30\%) showed a favorable outcome (CPC 1-2) compared to 49 (70\%) with an unfavorable outcome (CPC 3 - 5). NfL increased from CPC 1 to 5 (16.5 $\mathrm{pg} / \mathrm{ml}$ to $641 \mathrm{pg} / \mathrm{ml}, \mathrm{p}<0.001$ ). NfL alone performed moderately well with an area under the ROC (AUROC) of $79.4 \%$. Prediction was significantly improved by combination of $\mathrm{NfL}$ with the established best performing model $(F=6.83, p=0.01)$ with an AUROC to $89.7 \%$ ( $p$ for comparison $=0.017)$.

Conclusion:

The combination of $\mathrm{NfL}$ with other plasma and clinical markers is superior to that of either model alone and achieves a very good AUROC in this relatively small sample.

Trial registration: ClinicalTrials.gov NCT01960699. Registered 08 October 2013.

\section{Background}

Out-of-hospital cardiac arrest (OHCA) is a life-threatening event and intensive efforts have increased the number of patients that survive the initial event [1]. However, at the same time the rates of patients that can be discharged from hospital or the intensive care unit (ICU) with favorable neurological function, i.e. functional independence, does not expand at the same rate [2]. Consequently, the need for early and accurate prognostication of neurological outcome is greater than ever before. This is compounded by the fact that more recently, patients are treated with hypothermia and require sedation and frequently muscle relaxants for several days, and neurological assessment must be postponed [3]. As a result, many patients survive to reach a state of minimal consciousness and indeed a large proportion of deaths after OHCA can be attributed to active withdrawal of therapy [4]. Research into prediction of neurological 
outcome after OHCA is ongoing since cardiopulmonary resuscitation was first introduced. Ideally, such a predictor should be easily obtainable in a comatose patient and as such focus has been placed on serum markers of neurological damage, such as neuron specific enolase (NSE) or protein S-100B, but both markers have failed to reach adequate reliability in clinical use [5]. Lately, advances in detection technology have expanded the spectrum to include neurofilament light chain $(\mathrm{NfL})$ in serum. This has been shown to provide high discriminatory and predictive ability and can be measured with comparable reliability in serum and plasma $[6,7]$. We have recently shown that a model combining several clinical factors with four proteins, a-enolase, 14-3-3 protein $\zeta / \delta$, cofilin-1, and heat shock cognate $71 \mathrm{kDa}$ protein, that were identified through a semi-targeted proteomics approach can significantly improve prediction in this population [8]. The aim of this analysis was thus to assess the prognostic value of NfL to predict an unfavorable neurological outcome in an established cohort of OHCA survivors and to compare it to the existing best-performing multimarker model.

\section{Methods}

\section{Design and patients included}

This is a post-hoc analysis of a prospectively collected single-center study that included patients between October 2013 and May 2016. We recruited consecutive patients with OHCA that were treated at the emergency department of the Medical University of Vienna. Patients were included with a Glasgow coma scale score of 3 at admission and when the reason for cardiac arrest was either cardiac, respiratory, hemodynamic or metabolic. Patients were excluded when there was a history of previous cardiac arrest, neurological disorders or central nervous system neoplasms, psychiatric illness, substance abuse including alcohol, and the ongoing use of psychotropic medications. Patients were treated according to guidelines at the time of enrolment. Importantly, all patients were treated with targeted temperature management either before or upon arrival in the ED. For detailed information on the procedures and inclusion criteria please refer to [8]. Additional patient baseline information and laboratory assessments have been provided in subsequent publications $[9,10]$.

\section{Outcome}

The outcome was recorded at the time of transfer from ICU and rated on the 5-point cerebral performance category (CPC) [11] scale by an experienced neurologist (SS). Favorable neurological outcome was defined as a CPC of 1 or 2, while unfavorable neurological outcome was a CPC of 3-5.

\section{Handling of blood samples}

Blood samples were drawn 48 hours after OHCA. Samples were placed on ice after drawing, processed for the generation of platelet-poor plasma and then stored at $-80^{\circ} \mathrm{C}$ until further processing. 


\section{Analysis of biomarkers}

Neuron-specific enolase (NSE) and protein S100-B (S100B) lev-els were determined according to the local laboratory's standard procedure.

For NfL measures, samples were thawed for 60 minutes and were analyzed by an investigator blinded to any patient information using the Simoa Nf-light kits and consumables in the Simoa SR-X Analyzer (Quanterix, Lexington, MA, USA) [12]. The assay was performed according to the manufacturer's instructions and protocol. Briefly, thawed samples and calibrators were equilibrated to room temperature and dispensed in 96-well plates as duplicates. Samples and calibrators were incubated with detectors and paramagnetic bead solutions following instructions and incubated and washed using the Simoa microplate incubator and microplate washer. Sample readout and concentration analysis was calculated on the Quanterix SR-X Analyzer. The methods concerning proteomic analysis can be found elsewhere [8].

\section{Statistical methods}

Values are presented as means (+- standard deviation [SD]) or median (interquartile range [IQR]) as appropriate. Some variables were transformed due to high skewness, and missing values were imputed by chained equation [13]. Differences between groups were calculated using t-test for normally distributed variables, the Mann-Whitney-U test for non-normally distributed variables and the KruskalWallis rank sum test for comparison over multiple groups. Correlations were assessed using Spearman's rank correlation coefficient and, when necessary, corrected for multiple testing using the Bonferroni correction. Predictive models were constructed using logistic regression with unfavorable neurological outcome as the dependent variable. Model 1 (M1) consisted of NfL values alone. Model 2 (M2) was established to be the best performing combination by employing a statistical multi-step approach of ridge- and LASSO-regression to optimize performance [8]. M2 includes four proteins (heat shock protein C,14-3-3 protein, cofilin-1, and Enolase A) along with the clinical parameters age, the presence of a shockable rhythm at first analysis of rhythm, witnessed cardiac arrest (i.e. immediate start of chest compressions), the time until return of spontaneous circulation (ROSC), and hemoglobin, lactate and $\mathrm{pH}$ at admission. Model 3 (M3) combines M1 and M2. The benefit of adding NfL to M2 was analyzed using Wald test and the likelihood-ratio test.

The performance of a model to predict an unfavorable neurological outcome was assessed by calculating the area under the receiver operating characteristic curve (AUROC) and compared using DeLong's test for correlated AUC curves [14] and calculated using the $p R O C$ package for $\mathrm{R}$ [15]. Since the prediction of unfavorable neurological outcome comes with most serious consequences, we evaluated the sensitivity of the models at a specificity of $95 \%$. Additionally, optimal cut-offs were calculated using the Youden's J-statistic [16].

Calculations were performed using R (Version 4.0.2 R Foundation for Statistical Computing, Vienna, Austria.), a p-value of $<0.05$ was considered to be significant. 


\section{Results \\ Patients}

We initially enrolled 96 individuals who were successfully resuscitated after an OHCA and treated at our institution between 2013 and 2016. Sufficient plasma samples were available in 70 patients who were included in this analysis. The median age at the time of OHCA was 56.5 years (interquartile range [IQR] 47.5-68) and $24.3 \%$ were female. More details on the baseline characteristics can be found in Table 1. 


\begin{tabular}{|c|c|c|c|}
\hline & $\begin{array}{l}\text { Favorable neurological } \\
\text { outcome }\end{array}$ & $\begin{array}{l}\text { Unfavorable neurological } \\
\text { outcome }\end{array}$ & $\begin{array}{l}\mathrm{p}- \\
\text { value }\end{array}$ \\
\hline Number of patients & $21(30 \%)$ & $49(70 \%)$ & \\
\hline Female & $5(23.8 \%)$ & $12(24.5 \%)$ & 1 \\
\hline Age, years & $56[43,66]$ & $60[49,70]$ & 0.189 \\
\hline Cardiac arrest witnessed & $19(90 \%)$ & 37 (75\%) & 0.268 \\
\hline First rhythm shockable & $19(90 \%)$ & $35(71 \%)$ & 0.195 \\
\hline Time until ROSC, min. & $27.00[18.00,33.00]$ & $25.50[14.75,38.75]$ & 0.922 \\
\hline $\begin{array}{l}\text { Epinephrine administered, } \\
\text { mg }\end{array}$ & $3.00[1.00,4.00]$ & $4.00[2.75,5.00]$ & 0.318 \\
\hline \multicolumn{4}{|l|}{ Medical History } \\
\hline Diabetes & $2(9.5 \%)$ & $7(14.3 \%)$ & 0.876 \\
\hline Hypertension & $6(28.6 \%)$ & $20(40.8 \%)$ & 0.483 \\
\hline Smoking & $8(33.3 \%)$ & $19(38.8 \%)$ & 0.871 \\
\hline Chronic heart failure & $2(9.5 \%)$ & $5(10.2 \%)$ & 1 \\
\hline Coronary artery disease & $3(14.3 \%)$ & $8(16.3 \%)$ & 1 \\
\hline COPD & $2(9.5 \%)$ & $6(12.2 \%)$ & 1 \\
\hline \multicolumn{4}{|l|}{$\begin{array}{l}\text { Laboratory values at } \\
\text { admission }\end{array}$} \\
\hline $\mathrm{pH}$ & $7.23[7.17,7.29]$ & $7.21[7.11,7.26]$ & 0.405 \\
\hline Lactate & $7.30[5.20,9.00]$ & $7.50[5.10,10.30]$ & 0.929 \\
\hline p02 & $64.00[42.00,251.00]$ & $40.80[20.40,150.00]$ & 0.063 \\
\hline $\mathrm{pCO} 2$ & $15.60[7.40,23.80]$ & $16.00[10.10,25.90]$ & 0.568 \\
\hline Bicarbonate & $8.30[4.70,10.00]$ & $8.70[5.90,10.80]$ & 0.457 \\
\hline Base excess & $9.40[6.00,12.30]$ & $9.80[5.90,12.90]$ & 0.815 \\
\hline Hemoglobin & $14.60[13.40,15.30]$ & $14.40[12.60,15.40]$ & 0.644 \\
\hline Neuron specific enolase & $25.70[14.80,47.10]$ & $30.80[15.60,81.90]$ & 0.216 \\
\hline
\end{tabular}

ROSC - return of spontaneous circulation; COPD - chronic obstructive pulmonary disease; p02/pCO2 partial pressures. Values are given as $n(\%)$, mean (standard deviation) or median [interquartile range] 


\begin{tabular}{|lccc|}
\hline & $\begin{array}{l}\text { Favorable neurological } \\
\text { outcome }\end{array}$ & $\begin{array}{l}\text { Unfavorable neurological } \\
\text { outcome }\end{array}$ & $\begin{array}{l}\text { p- } \\
\text { value }\end{array}$ \\
\hline Protein S-100B & $0.08[0.06,0.09]$ & $0.14[0.08,0.36]$ & 0.002 \\
\hline $\begin{array}{l}\text { ROSC - return of spontaneous circulation; COPD - chronic obstructive pulmonary disease; } \mathrm{pO} 2 / \mathrm{pCO} \text { - } \\
\text { partial pressures. Values are given as n (\%), mean (standard deviation) or median [interquartile range] }\end{array}$ \\
\hline
\end{tabular}

\section{NfL levels}

The median level of NfL at 48 hours after OHCA was 66.58 (IQR 16.86-989.53) pg/ml. The levels were skewed heavily to the left with a minimum value of $4.28 \mathrm{pg} / \mathrm{ml}$ and a maximum level of $61715.58 \mathrm{pg} / \mathrm{ml}$. After log2-transformation and correction for multiple testing, $\mathrm{NfL}$ levels were correlated significantly and positively with two clinically established plasma markers: neuron specific enolase (NSE) (Spearman's rho $=0.44, \mathrm{p}<0.001$; and protein S-100B (rho $=0.54, \mathrm{p}<0.001 ;$ Fig. 1 ).

\section{Neurological outcome and NfL}

At discharge from the intensive care unit, 21 patients $(30 \%)$ had a favorable neurological outcome defined as a cerebral performance category (CPC) score of either 1 (17 patients [24\%]) or 2 (4 patients [6\%]), and 49 patients (70\%) had an unfavorable neurological outcome, i.e. a CPC of 3 (21 patients [30\%]), 4 (11 patients [16\%]) or 5 (17 patients [24\%]). NfL levels increased significantly with CPC score, with a median level of 16.5 (IQR 9.35-21.4) pg/ml for CPC 1 compared to a median level of 641 (IQR 1972173 ) $\mathrm{pg} / \mathrm{ml}$ for the CPC 5 group ( $\mathrm{p}$ for all groups $<0.0001$ ). Comparing favorable outcome vs. unfavorable outcome, we find a median of 18.5 (IQR 11.3-28.2) pg/ml for favorable outcome compared to a median of 297 (IQR 39.7-1572) pg/ml for unfavorable neurological outcome $(p<0.0001$; Fig. 2$)$.

\section{Predictive performance of NfL (M1)}

$\mathrm{NfL}$ levels measured in serum at 48 hours after OHCA showed a moderately favorable performance for predicting unfavorable neurological outcome with an area under the ROC curve (AUROC) of $79.4 \%$ (95\% confidence interval [Cl]: 68.3-90.5). The sensitivity was relatively high with $61.2 \%$ at a specificity of $95 \%$. The optimal cut-off calculated with the Youden index had a specificity of $85.7 \%$ and a sensitivity of $71.4 \%$. AUROC for NfL was significantly greater than that for NSE (AUROC of $59.3 \%, p=0.02$ ) and numerically greater than that for protein S-100B (AUROC of $73.1 \%, p=0.4$ ).

\section{Comparison to multimarker model M2 and M3}

In the cohort analyzed here, M2 performs moderately well to predict an unfavorable neurological outcome with an AUROC of $81.3 \%$ (95\% Cl: $71.4-91.3)$ but showed a lower sensitivity than $\mathrm{NfL}$ at a specificity of 
$95 \%$ with $53 \%$. The optimal cutoff showed a specificity of $81 \%$ with a sensitivity of $69.4 \%$.

We then added the parameters of the previously established model M2 to the NfL model and termed this combination M3. The addition of $\mathrm{NfL}$ significantly improved the performance of $\mathrm{M} 2$ (Wald test: $\mathrm{F}=6.83$, $p=0.01$; likelihood-ratio $=11.16, p<0.01$ ) and M3 performed well to predict an unfavorable neurological outcome with an AUROC of $89.7 \%$ (95\% Cl: 81.7-97.7). Compared to the existing models, M3 had a numerically greater AUROC than M2 $(89.7 \%$ vs. $81.3 \%, p=0.054)$ and a significantly greater AUROC than M1 $(89.7 \%$ vs. $79.4 \%, p=0.017)$. The combined model M3 showed a sensitivity of $67.4 \%$ at $95 \%$ specificity and the optimal cut-off had a specificity of $90.5 \%$ and a sensitivity of $81.6 \%$.

\section{Discussion}

Neurofilament light chain (NfL) has emerged as the most promising blood marker to predict neurological survival after cardiac arrest. In a preceding work, we constructed a prediction model consisting of clinical and plasma biomarkers in combination with the four proteins, a-enolase, 14-3-3 protein $\zeta / \delta$, cofilin- 1 , and heat shock cognate $71 \mathrm{kDa}$ protein, that were measured by high-throughput proteomics. Here, we used a high-sensitive assay to assess the ability of $\mathrm{NfL}$ to predict unfavorable neurological outcome in this previously established cohort of OHCA survivors. We found that it performs moderately well with an AUROC of $79.4 \%$. The combination of $\mathrm{NfL}$ with the established model, however, significantly improved the model and yielded an AUROC of $90 \%$.

Predicting neurological outcome after cardiac arrest is intrinsically a high-risk endeavor, as the choice is often between continuing or withdrawing life supporting care. Information on the neurological outcome can inform this decision, but must do so with utmost caution. Accordingly, a multimarker approach that includes clinical and laboratory parameters is recommended [17]. The optimal time-point to assess outcome is unclear, however, targeted temperature management and the ensuing sedation has necessarily increased the interval for clinical prediction and impedes neurological testing. As such, objective biomarkers need to be introduced and preferably aid prognostication as early as 24 to 48 hours after the initial event.

We found high NfL concentrations 48 hours after OHCA to be significantly associated with unfavorable neurological outcome. In fact, there was an almost exponential rise of NfL levels with increasing scores on the CPC scale. This is in line with a larger trial of over 700 patients [6] where the AUROC for NfL reached a very high $94 \%$. In our cohort, NfL showed a much lower value. This could be due to a combination of sample size and different inclusion criteria, as the trial by Moseby-Knappe et al. was a substudy of a randomized trial to study targeted temperature measurement that included only patients with presumed cardiac causes of OHCA and measured NfL in serum [18]. Generally, serum and plasma $\mathrm{NfL}$ concentrations correlate well, but plasma typically yields about $75 \%$ the concentration of serum NfL [19]. Of note, we measured plasma NfL levels of $5026 \mathrm{pg} / \mathrm{ml}$ in a patient who survived to excellent neurological function with a CPC of 1 . This is in excess of the cut-offs suggested in the larger trial, 
beyond which there was not a single case with favorable outcome found. This emphasizes that a singlemarker model is vulnerable to these outliers.

We found that $\mathrm{NfL}$ correlated well and with similar strength with the two clinically established markers of neuronal damage, NSE and protein S100-B, which are found in neurons and astrocytes, respectively. However, the AUROC for NfL exceeded both of the others, albeit only statistically significant compared to NSE which performed poorly with an AUROC of $59 \%$ in this cohort. While both markers are not suitable to predict outcome on their own, they could be integrated into a multi-modal approach [20]. In the cohort studied here, S100-B performed moderately well with an AUROC of 73\%. In a previous work, we combined clinical information, laboratory values and performed a semi-targeted proteomic approach in which we focused on brain derived proteins [8]. Twenty-four proteins were present both in brain tissue from autopsy samples and patients that survived OHCA and were included in the analysis. A multi-step regression approach ultimately identified a model consisting of several clinical and laboratory markers, including S100B but not NSE, and four proteins that were previously not used for prediction. We showed that these markers improved prediction of poor neurological outcome. In this study we combined this model with $\mathrm{NfL}$ measured at 48 hours after cardiac arrest and improved the AUROC to predict poor neurological survival significantly. Ultimately, a combination of blood-based markers that reflect damage to the central nervous system show the most promise in this regard. Currently, prediction modeling benefits from the inclusion of other variables that are linked to the duration of poor cerebral perfusion, such as time until ROSC and pH at admission. However, further analysis of our model or others in larger, multicenter cohorts could pave the way towards an exclusively clinical parameter and blood-based risk prediction.

\section{Limitations}

This study is inherently limited by its design as post-hoc analysis of a prospective trial. The primary trial was intended as a pilot study and the complex and expensive proteomic analysis limited the sample size. A further limitation is that it is a single-center analysis, which might reduce generalizability. However, it allowed the inclusion of a homogenous sample and high quality of sample processing and storage. Lastly, the trial was observational and should be used to generate hypotheses.

\section{Conclusions}

This study confirms previous observations of NfL concentrations being substantially elevated in patients with poor neurological outcome after OHCA. NfL showed good predictive performance when measured 48 hours after the event. However, the addition of a previously established multimarker model significantly improved this model to achieve an AUROC of almost $90 \%$. Further studies including multi(bio)marker assessment appear warranted to confirm our findings.

\section{Declarations}




\section{Ethics approval and consent to participate}

The study was approved by the IRB of the Medical University of Vienna (EK 1740/2013) and the primary trial was registered with ClinicalTrials.gov on the 8th of October 2013 (NCT01960699, https://clinicaltrials.gov/ct2/show/NCT01960699). In case of awakening, written informed consent was retroactively obtained.

\section{Consent for publication}

Not applicable

\section{Availability of data and material}

The datasets during and/or analysed during the current study available from the corresponding author on reasonable request. The remaining authors have disclosed that they do not have any potential conflicts of interest.

\section{Competing interests}

Drs. Distelmaier, Wurm, and Adlbrecht disclosed that they have a patent pending for the previously identified biomarkers.

\section{Funding}

This study was supported by the Medical Scientific Fund of the Mayor of the City of Vienna \#19061 (2019, to CA), the Anniversary Fund of the Oesterreichische Nationalbank OENB \#15959 (2014, to CA) and a Research grant of the Austrian Society of Cardiology (2014, to CA). None of the funding bodies were involved in the design, collection, analysis or interpretation of the data nor the writing of this manuscript.

\section{Authors' contributions}

RW, PR, SS, CA and KD were involved in conception and design of this trial; RW, $H A, B M, A B, M P$ and PA were involved in acquisition of the samples and data, RW performed the statistical analysis and RW, CG, $C A$ and $K D$ were involved in the interpretation of the data, RW drafted the manuscript and PR, $P H, F S, G H$, $\mathrm{CG}, \mathrm{CA}$ and $\mathrm{KD}$ provided substantial revisions.

\section{Acknowledgements}


Not applicable.

\section{References}

1. NehmeZ,AndrewE,BernardS,HaskinsB,SmithK.Trends in survival from out-of-hospital cardiac arrests defibrillated by paramedics, first responders and bystanders.Resuscitation.2019;143:85-91.

2. Buick JasonE.,Drennan lanR.,Scales DamonC.,Brooks StevenC.,ByersAdams,CheskesSheldon,etal.Improving Temporal Trends in Survival and Neurological Outcomes After Out-of-Hospital Cardiac Arrest.Circ Cardiovasc Qual Outcomes.2018;11:e003561.

3. GolanE,BarrettK,AlaliAS,DuggalA,JichiciD,PintoR,etal.Predicting neurologic outcome after targeted temperature management for cardiac arrest: systematic review and meta-analysis.Crit Care Med.2014;42:1919-1930.

4. MulderM,GibbsHG,SmithSW,DhaliwaIR,ScottNL,SprenkleMD,etal.Awakening and withdrawal of lifesustaining treatment in cardiac arrest survivors treated with therapeutic hypothermia*.Crit Care Med.2014;42:2493-2499.

5. ZellnerT,GärtnerR,SchopohlJ,AngstwurmM.NSE and S-100B are not sufficiently predictive of neurologic outcome after therapeutic hypothermia for cardiac arrest.Resuscitation.2013;84:13821386.

6. Moseby-KnappeM,MattssonN,NielsenN,ZetterbergH,BlennowK,DankiewiczJ,etal.Serum Neurofilament Light Chain for Prognosis of Outcome After Cardiac Arrest.JAMA Neurol.2019;76:6471.

7. HviidCVB,KnudsenCS,ParknerT.Reference interval and preanalytical properties of serum neurofilament light chain in Scandinavian adults.Scand J Clin Lab Invest.2020;80:291-295.

8. DistelmaierK,MuqakuB,WurmR,ArfstenH,SeidelS,KovacsGG,etal.Proteomics-Enriched Prediction Model for Poor Neurologic Outcome in Cardiac Arrest Survivors.Crit Care Med.2020;48:167-175.

9. OndracekAS,HofbauerTM,WurmR,ArfstenH,SeidIV,FrühA,etal.Imbalance between plasma doublestranded DNA and deoxyribonuclease activity predicts mortality after out-of-hospital cardiac arrest.Resuscitation.2020;151:26-32.

10. FrühA,GoliaschG,WurmR,ArfstenH,SeidelS,GalliL,etal.Gastric regurgitation predicts neurological outcome in out-of-hospital cardiac arrest survivors. Eur $\mathrm{J}$ Intern Med.2020.doi:10.1016/j.ejim.2020.08.010

11. Hypothermia after Cardiac Arrest Study Group.Mild therapeutic hypothermia to improve the neurologic outcome after cardiac arrest.N Engl J Med.2002;346:549-556.

12. RissinDM,KanCW,CampbellTG,HowesSC,FournierDR,SongL,etal.Single-molecule enzyme-linked immunosorbent assay detects serum proteins at subfemtomolar concentrations.Nat Biotechnol.2010;28:595-599.

13. vanBuurenS,Groothuis-OudshoornK.mice: Multivariate Imputation by Chained Equations in R.Journal of Statistical Software, Articles.2011;45:1-67. 
14. DeLongER,DeLongDM,Clarke-PearsonDL.Comparing the areas under two or more correlated receiver operating characteristic curves: a nonparametric approach.Biometrics.1988;44:837-845.

15. RobinX,TurckN,HainardA,TibertiN,LisacekF,SanchezJ-C,etal.pROC: an open-source package for R and $S+$ to analyze and compare ROC curves.BMC Bioinformatics.2011;12:77.

16. YoudenWJ.Index for rating diagnostic tests.Cancer.1950;3:32-35.

17. NolanJP,SoarJ,CariouA,CronbergT,MoulaertVRM,DeakinCD,etal.European Resuscitation Council and European Society of Intensive Care Medicine 2015 guidelines for post-resuscitation care.Intensive Care Med.2015;41:2039-2056.

18. NielsenN,WetterslevJ,al-SubaieN,AnderssonB,Bro-Jeppesen J,BishopG,etal.Target Temperature Management after out-of-hospital cardiac arrest-a randomized, parallel-group, assessor-blinded clinical trial-rationale and design.Am Heart J.2012;163:541-548.

19. SejbaekT,NielsenHH,PennerN,PlavinaT,MendozaJP,MartinNA,etal.Dimethyl fumarate decreases neurofilament light chain in CSF and blood of treatment naïve relapsing MS patients.J Neurol Neurosurg Psychiatry.2019[cited 27 Oct 2020].doi:10.1136/jnnp-2019-321321

20. WangC-H,ChangW-T,SuK-I,HuangC-H,TsaiM-S,ChouE,etal.Neuroprognostic accuracy of blood biomarkers for post-cardiac arrest patients: A systematic review and metaanalysis.Resuscitation.2020;148:108-117.

\section{Figures}




\section{Figure 1}

Plasma levels of NfL vs NSE / Protein S100B

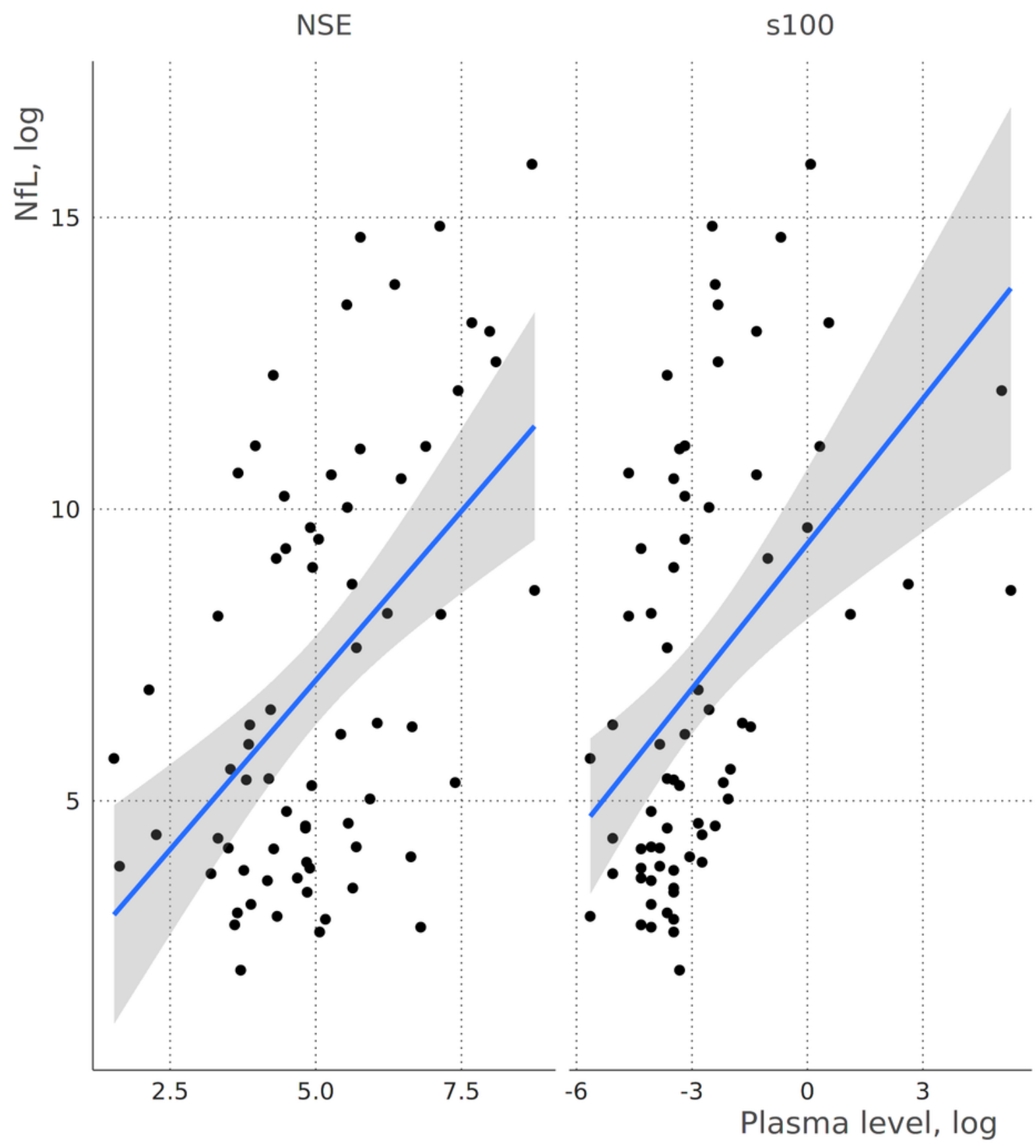

Figure 1

Correlation of plasma levels of neurofilament light chain ( $\mathrm{NfL}$ ) with plasma levels of two markers of neuronal damage, protein S100B and neuron specific enolase. 


\section{Figure 2}

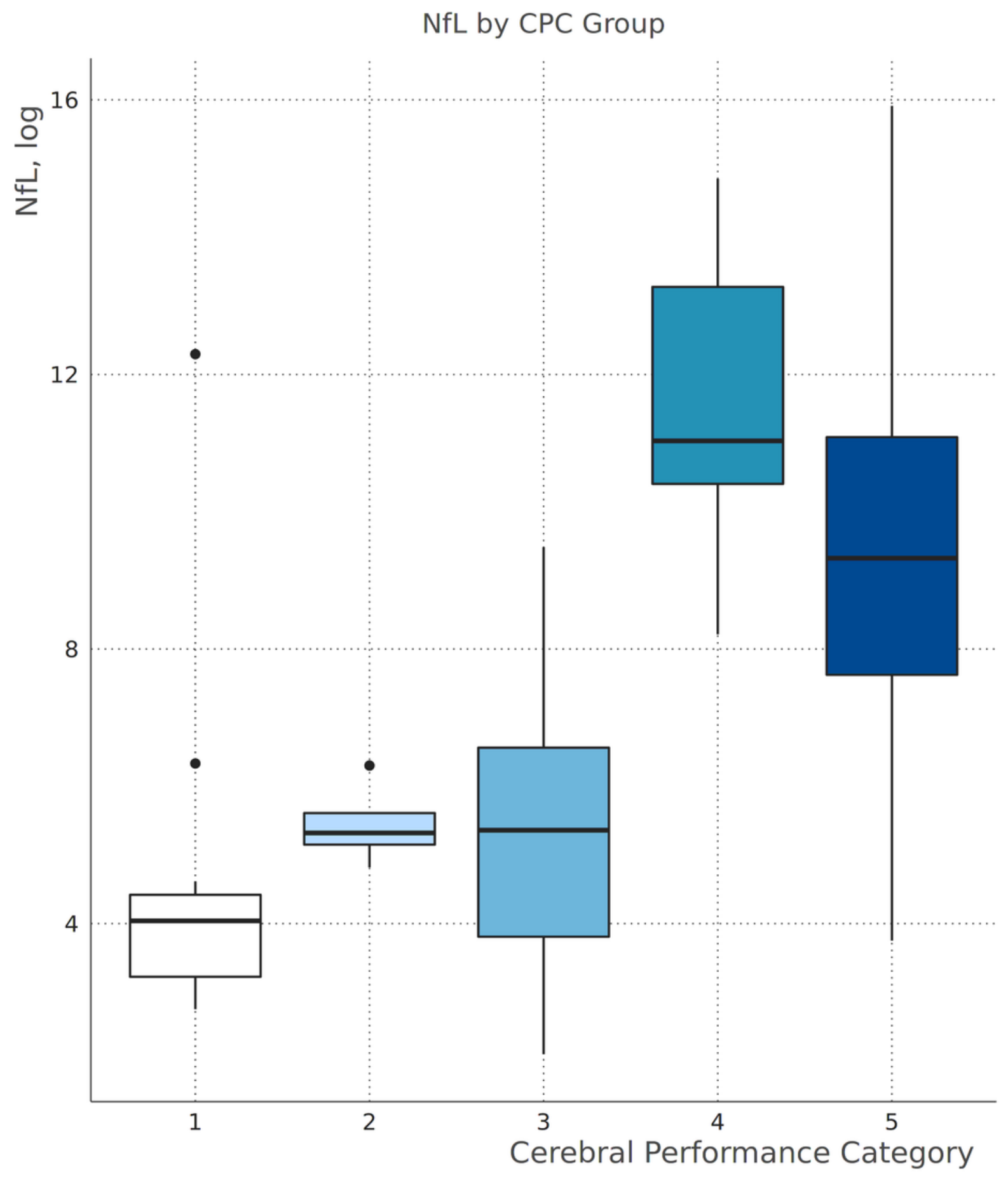

Figure 2

Boxplot showing plasma levels of neurofilament light chain $(\mathrm{NfL})$ grouped by neurological outcome measured on the five-point cerebral performance category scale (CPC) at discharge from the intensive care unit. 
Figure 3

ROC Comparison

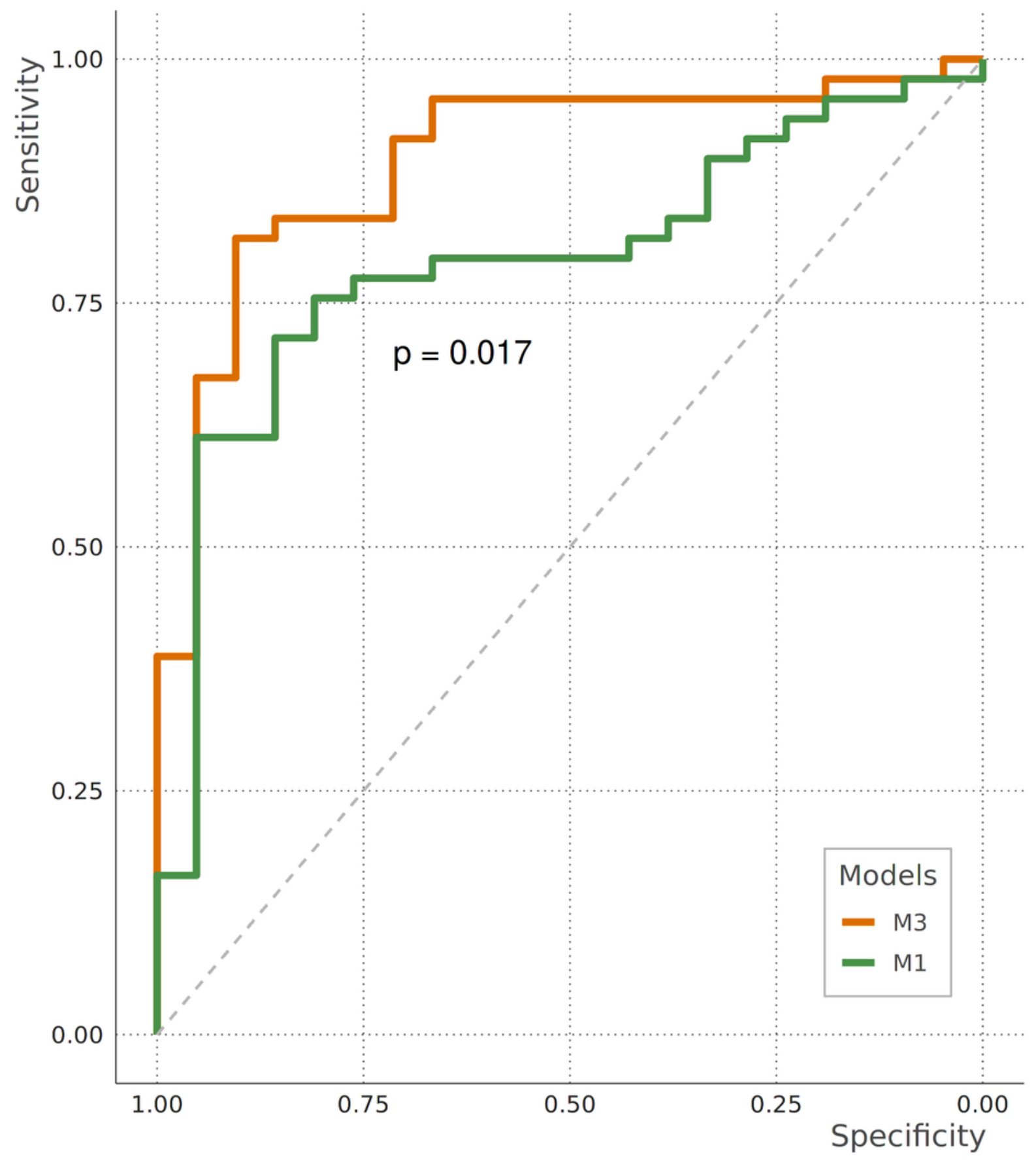

Figure 3

Area under the receiver operator curve (ROC) to compare the performance of neurofilament light chain (NfL, M1) to a multimarker model including NfL (M3) for predicting neurological outcome.. 